\title{
EVALUATION OF VITAMIN D SERUM LEVELS IN PATIENTS WITH INSOMNIA
}

\author{
MARIA JAI JOSEPH ${ }^{1}$, KARTHIK V P ${ }^{2 *}$ \\ ${ }^{1}$ Sri Ramachandra Medical College, Chennai, Tamil Nadu, India. ${ }^{2}$ Department of Pharmacology, Sri Ramachandra Medical College, Chennai, \\ Tamil Nadu, India. Email: dr_karthikvp@yahoo.in
}

Received: 07 June 2018, Revised and Accepted: 09 July 2018

ABSTRACT

Objectives: The primary objective of the study was to evaluate the link existing between insomnia and low levels of Vitamin D and the secondary objective was to confirm a link between age and insomnia.

Methods: The study assessed the Vitamin D serum levels in 50 subjects with insomnia (diagnosed using Pittsburgh Sleep Quality Index [PSQI]) from the outpatient clinic, Department of General Medicine of Sri Ramachandra Medical Center. All subjects fulfilled the eligibility criteria and also signed the informed consent form provided in patient's native language for their convenience. With all aseptic precautions, $5 \mathrm{ml}$ of blood was collected from the subjects for tyhe measurement of 25-OH Vitamin-D total levels using fully automated chemiluminescent immunoassay. Statistical analysis was performed using the Statistical Package for the Social Sciences (SPSS 1 7.1). Quantitative variables will be expressed as mean \pm standard deviation (SD), and qualitative variables will be expressed as percentage values.

Results: All the patients who are insomniacs were deficient of Vitamin D. Of this, $80 \%$ were in deficient state (i.e.,> $20 \mathrm{ng} / \mathrm{ml}$ ) and the rest $20 \%$ were in the insufficient state (i.e., $20-30 \mathrm{ng} / \mathrm{ml}$ ). Most of the insomniacs were younger adults. As shown in the graph, 27 patients were in between the age group of 20 and 30 years, 13 patients of 50 patients were between 30 and 40 years of age, 4 patients between 40 and 50 years of age, 4 patients between 50 and 60 years of age, and 2 patients were of age between 60 and 70 years.

Conclusion: Our results concluded that insomnia has a direct link with Vitamin D deficiency. Male insomniacs who were Vitamin D deficient were $40 \%$ more than female insomniacs with Vitamin D deficiency. It was noted that the incidence of increased body mass index and systolic blood pressure was seen among Vitamin D-deficient insomniacs. Insomnia was more prevalent among younger age groups rather than older adults. Global PSQI value evaluated among all Vitamin D-deficient patients was $>5$, which indicated poor sleep quality.

Keywords: Vitamin D, Insomnia, Pittsburgh Sleep Quality Index.

(C) 2018 The Authors. Published by Innovare Academic Sciences Pvt Ltd. This is an open access article under the CC BY license (http://creativecommons. org/licenses/by/4. 0/) DOI: http://dx.doi.org/10.22159/ajpcr.2018.v11i11.27776

\section{INTRODUCTION}

Insomnia, also known as sleeplessness, is a sleep disorder where people have trouble sleeping. They may have difficulty falling asleep or staying asleep as long as desired [1,2]. Insomnia is typically followed by daytime sleepiness, low energy, irritability, and a depressed mood. It may result in an increased risk of motor vehicle collisions, as well as problems focusing and learning. Insomnia can be short term, lasting for days or weeks, or long term, lasting more than a month.

Insomnia can occur independently or as a result of another problem. Conditions that can result in insomnia include psychological stress, chronic pain, heart failure, hyperthyroidism, heartburn, restless leg syndrome, menopause, certain medications, and drugs such as caffeine, nicotine, and alcohol. Other risk factors include working night shifts and sleep apnea. Diagnosis is based on sleep habits and an examination to look for underlying causes. A sleep study may be done to look for underlying sleep disorders. Sleep hygiene and lifestyle changes are typically the first treatment for insomnia. Sleep hygiene includes a consistent bedtime, exposure to sunlight, a quiet and dark room, and regular exercise. While sleeping pills may help, they are associated with injuries, dementia, and addiction. Medications are not recommended for more than 4 or 5 weeks. The effectiveness and safety of alternative medicine are unclear.

Between $10 \%$ and $30 \%$ of adults have insomnia at any given point in time and up to half of the people have insomnia in a given year. About $6 \%$ of people have insomnia that is not due to another problem and lasts for more than a month. People over the age of 65 are affected more often than younger people. Females are more often affected than males.
Vitamin D deficiency can be caused by a number of factors including insufficient cutaneous synthesis (due to limited sunlight exposure or aging, inadequate intake and absorption of vitamin D, obesity, or darker skin) [3]. Elderly people (due to decreased outdoor activity), individuals with darker skin tone (as melanin absorbs ultraviolet radiation), and pregnant/lactating women and children are at increased risk of Vitamin D deficiency.

Serum $25(\mathrm{OH})$ Vitamin D is the major circulating metabolite of Vitamin D and reflects Vitamin D inputs from cutaneous synthesis and dietary intake. Serum $25(\mathrm{OH})$ is the standard clinical measure of Vitamin D status [4]. Vitamin D deficiency is defined as a level $<20 \mathrm{ng} / \mathrm{ml}$ and Vitamin D insufficiency is a level $<30 \mathrm{ng} / \mathrm{ml}$.

Insomnia maybe due to physical discomfort, psychological factors, and fluctuations in metabolic control, and many of these factors are also associated with Vitamin D deficiency. Quality of life is affected, and coping with the disease is made difficult by insomnia [5].

Vitamin D deficiency subjects have high levels of tumor necrosis factor-alpha (TNF- $\alpha$ ), an inflammatory marker which is implicated in sleepiness associated with obstructive sleep apnea (OSA) [6]. The rise in inflammatory marker may also contribute to disturbed sleep in some individuals, suggesting the possibility that Vitamin D deficiency could represent a condition which predisposes an individual to the development of pathological degrees of central nervous system (CNS)induced sleep disorder.

Vitamin D plays a major role in the pathogenesis and prevention of insomnia. Prevention of Vitamin D deficiency may become, in future, 
a strategy to prevent the development of insomnia and avoid its deleterious consequences on health. It is very important to assess the Vitamin D status because the effective detection and treatment of inadequate Vitamin D levels are easy and cost-effective therapy which could improve individuals sleep pattern which in turn can improve long-term health outcomes and quality of life. The aim of the study is to assess the Vitamin D serum levels in patients with Insomnia.

\section{METHODS}

\section{Study design}

This was a prospective, cross-sectional, analytical study

\section{Sample size}

The sample size was 50 .

\section{Study population}

The study assessed the Vitamin D serum levels in 50 subjects with insomnia (diagnosed using Pittsburgh Sleep Quality Index [PSQI]) from the outpatient clinic, Department of General Medicine of Sri Ramachandra Medical Center. All subjects fulfilled the eligibility criteria and also signed the informed consent form provided in patient's native language for their convenience. With all aseptic precautions, $5 \mathrm{ml}$ of blood was collected from the subjects for the measurement of Vitamin-D levels. This study was approved by the Ethical Committee.

The following criteria to be met by screened subjects.

\section{Inclusion criteria}

- Sex: Both male and female

- Age group: 20-70 years

- Subjects who provided a written informed consent

- Subjects were willing to cooperate with the study procedures.

\section{Exclusion criteria}

- Subjects with psychiatric disorders

- Subjects taking psychoactive drugs or stimulants, herbs, nicotine, cocaine, amphetamines, methylphenidate, modafinil, fluoroquinolone antibiotic drugs.

\section{Laboratory investigations}

- Serum 25-OH Vitamin D - Radioimmunoassay (RIA Kit method).

\section{Research tool}

In all subjects, quality of sleep was evaluated through the PSQI through the interview method. PSQI assesses subjective sleep quality over the past 4 weeks. The PSQI is a self-report questionnaire that assesses sleep quality and quantity over a 1 month period. The questionnaire consisted of 19 self-rated questions and 5 questions that were to be answered by bedmates or roommates. The latter questions are used only for clinical information and are not used in the scoring. 19 questions are categorized into 7 components, graded on a score that ranges from 0 to 3 . The PSQI components are as follows: Subjective sleep quality (C1), sleep latency (C2), sleep duration (C3), habitual sleep efficiency (C4), sleep disturbances (C5), use of sleeping medication (C6), and daytime dysfunction (C7). The sum of scores for these seven components yields one global score, which ranges from 0 to 21, where the highest score indicates worst sleep quality. A global PSQI $\geq 5$ has a diagnostic sensitivity of 89.6 and specificity of 86.5 in distinguishing "poor sleepers" (PSQI $\geq 5$ ) from "good sleepers" (PSQI <5) subjects. The design of the PSQI is such that the items and the component scores represent standard areas that clinicians usually focus on when patients report sleep problems.

\section{Statistical analysis}

A statistical analysis was performed using the SPSS 1 7.1. Quantitative variables will be expressed as mean $\pm \mathrm{SD}$, and qualitative variables will be expressed as percentage values.

\section{RESULTS}

All the patients who are insomniacs were deficient of Vitamin D. Of this, $80 \%$ were in deficient state (i.e., $>20 \mathrm{ng} / \mathrm{ml}$ ) and the rest $20 \%$ were in insufficient state (i.e., $20-30 \mathrm{ng} / \mathrm{ml}$ ). Most of the insomniacs were younger adults. As shown in the graph, 27 patients were in between the age group of 20 and 30 years, 13 patients of 50 patients were between 30 and 40 years of age, 4 patients between 40 and 50 years of age, 4 patients between 50 and 60 years of age, and 2 patients were of age between 60 and 70 years.

All the patients who are insomniacs were deficient of Vitamin D. Of this, $80 \%$ were in deficient state (i.e., $>20 \mathrm{ng} / \mathrm{ml}$ ) and the rest $20 \%$ were in insufficient state (i.e., $20-30 \mathrm{ng} / \mathrm{ml}$ ).

Most of the insomniacs were younger adults. As shown in the graph, 27 patients were in between the age group of 20 and 30 years, 13 patients of 50 patients were between 30 and 40 years of age, 4 patients between 40 and 50 years of age, 4 patients between 50 and 60 years of age, and 2 patients were of age between 60 and 70 years.

Most of the patients take more than $1 \mathrm{~h}$ to fall asleep from the time of them going to bed. 30 of our patients took more than $60 \mathrm{~min}$ and 16 of them took 31-60 min, 4 patients between 16 and $30 \mathrm{~min}$, and none fell asleep $<15 \mathrm{~min}$.

\section{DISCUSSION}

A total of 50 patients were assessed, and all the patients met the inclusion and exclusion criteria set for the study.

According to Verdoia et al. and Shiva Fajahih, lower Vitamin D levels are observed in females as compared to males [7]. This is in contrast to our study where lower Vitamin D levels are observed in males as compared to females (Fig. 1). This may be because most of the male patients these days are involved in sedentary occupations such as IT professionals, teachers, students, and housewife which prevent them from sun exposure because sunlight is the most common source of Vitamin D.

In our study, Vitamin D levels were markedly decreased in patients suffering from insomnia.All the 50 patients we assessed had Vitamin D deficiency. Of this, $80 \%$ are in deficient state (i.e., $>20 \mathrm{ng} / \mathrm{ml}$ ) and the rest $20 \%$ are in insufficient state (i.e., $20-30 \mathrm{ng} / \mathrm{ml}$ ) (Fig. 2).

The sleep quality was evaluated using the PSQI. As of the PSQI grading, a score of 5 or $>5$ is indicative of poor sleep quality. All the 50 patients (Fig. 8) who were assessed had a total PSQI score $>6$,

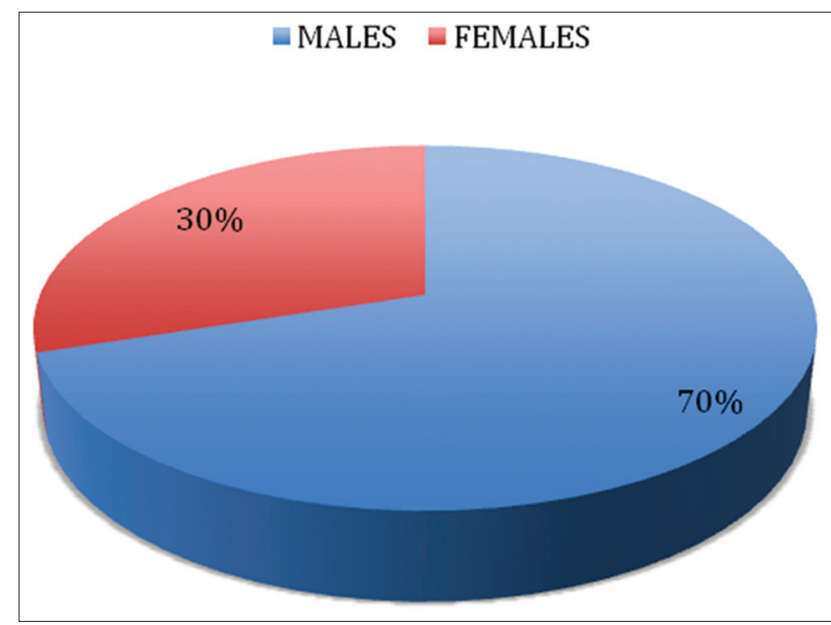

Fig. 1: Percentage of Vitamin $D$ deficiency in males and females having insomnia. In this study, male insomniacs who were Vitamin D deficient were $70 \%$ i.e., of 50 patients, 35 patients were males, whereas only 15 patients were females 


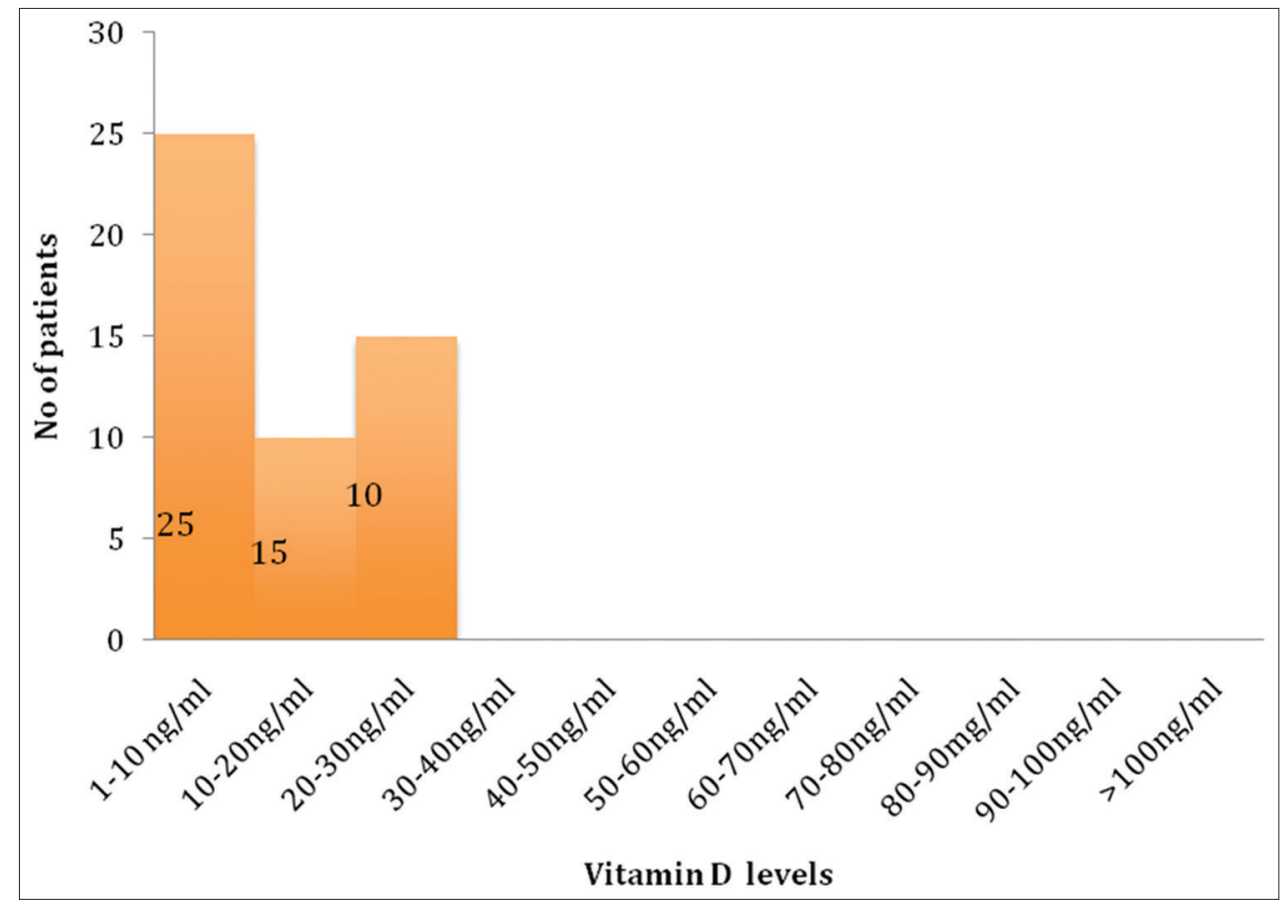

Fig. 2: Vitamin D levels in insomnia patients

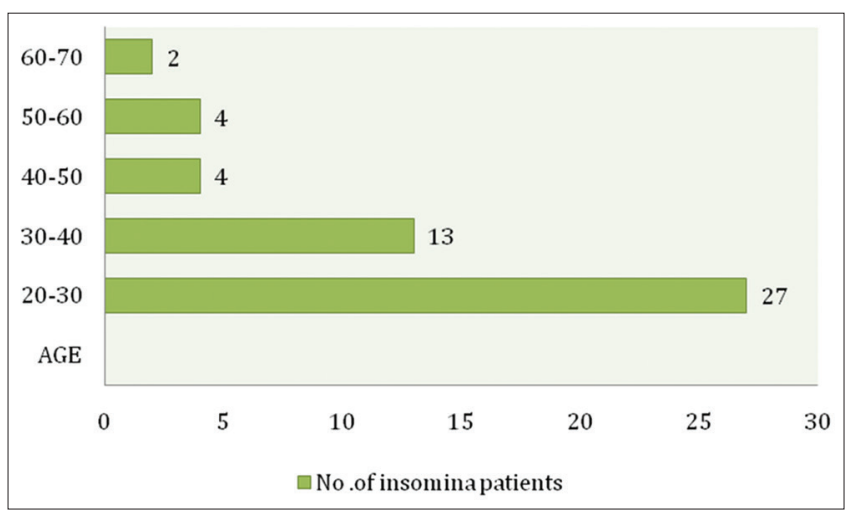

Fig. 3: Relation between age and incidence of insomnia

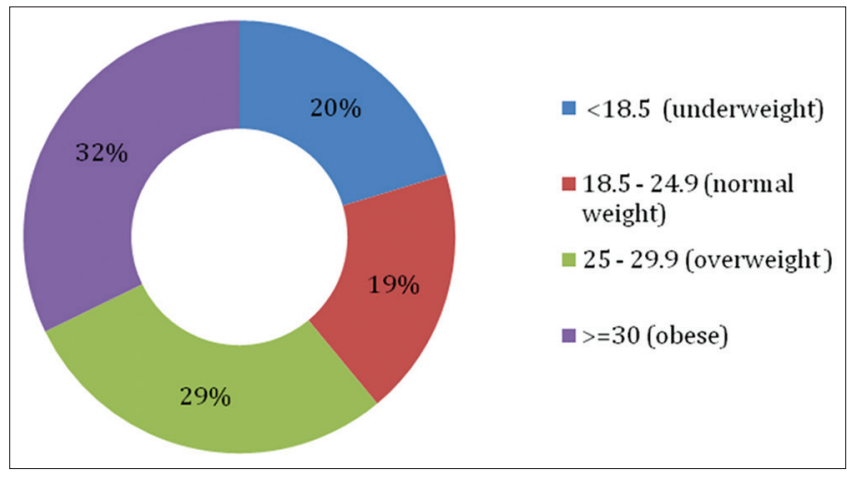

Fig. 4: Body mass index (BMI) and percentage of patients with insomnia having Vitamin D deficiency. With increasing BMI, a decrease in Vitamin D serum levels is observed in insomniacs. 12 patients were in the category of underweight, i.e., $<18.5 \mathrm{BMI}$, 11 patients belonged to normal weight category, and 17 and 19 were under overweight and obese categories, respectively

suggesting that all our patients are insomniacs (Fig. 7). A greater global PSQI score may mean that the person takes a longer time to fall asleep

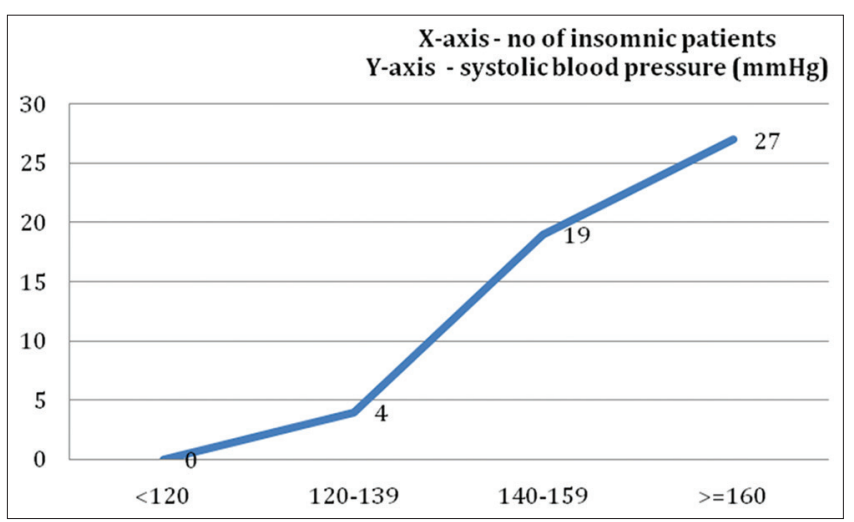

Fig. 5: Linear graph representing the relation between systolic blood pressure and the number of patients with insomnia having Vitamin D deficiency. Insomniacs with Vitamin D deficiency tend to have increased systolic blood pressure

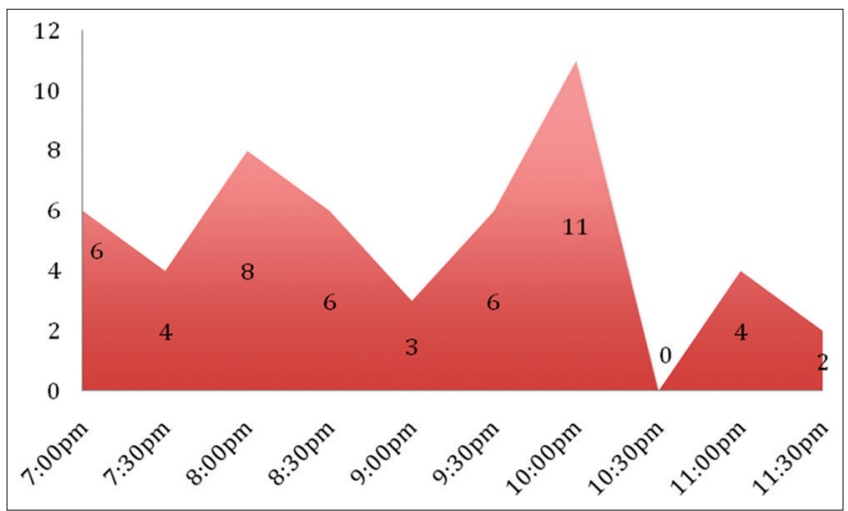

Fig. 6: Area graph plotting the number of insominic patients and their time of going to bed. The maximum number of patients goes to sleep by 10:00 PM (11 patients of 50). A slight increase (8 patients) is seen around 8:00 PM also 
or may indicate waking very early. A greater score can also mean that sleep is fragmented and restless, with many awakenings throughout the night (Table 1) [8,9].

Multiple factors likely contribute to poor sleep quality in Vitamin D deficiency subjects. Vitamin D has a direct neuroregulatory activity. Vitamin D receptor (VDR) and $25(\mathrm{OH}) \mathrm{D} 31$, a hydroxylase (catalyzes hydroxylation of calcidiol to calcitriol) are widely distributed throughout CNS. VDR gene polymorphisms have been associated with cognitive impairment and depressive symptoms which in turn are associated with poor sleep quality $[10,11]$. Further, Vitamin D is a potent biologic regulator of prostaglandin synthesis, the major prostanoid in the brain, and is the physiologic regulator of sleep. Vitamin D-deficient individuals have low levels of prostaglandin D2 which is attributed to poor sleep outcomes.

The immunomodulatory effect of Vitamin D has been related to recent evidence that inflammation may play a causal role in altered sleep pattern; Vitamin D has been shown to downregulate inflammatory mediators, such as nuclear factor-kB, which have been linked to sickness behavior, psychosocial stress, and depression.

Vitamin D deficiency subjects have high levels of TNF- $\alpha$, an inflammatory marker that is implicated in sleepiness associated with OSA. The rise in inflammatory marker may also contribute to disturbed sleep in some individuals, suggesting the possibility that Vitamin D deficiency could represent a condition which predisposes an individual to the development of pathological degrees of CNS-induced sleep disorder. Insomnia observed in individual with low Vitamin D levels is possible due to physical and physiological discomfort associated with Vitamin D deficiency.

Observing the graph plotted with age and incidence of insomnia (Fig. 3), most of the insomniacs were younger adults. As shown in the graph, 27 of our patients were in between the age group of 20 and 30, 13 of 50 were between 30 and 40 , 4 between 40 and 50 years of age, 4 between 50 and 60 years of age also, and 2 between 60 and 70 years. This is due to the increased usage of social media in which many younger adults have even become addicted to it in such a way that they are sleep deprived. Other reason would be the professional and financial stress. This is in contrast with the results by Zhang et al. and Roth, and in their studies, incidence of insomnia was increased with age $[12,13]$. The possible explanations given by them were that older adults experience a shift in the circadian rhythm that causes them to become sleepy in the early evening and to wake up too early in the morning. Other reasons would be like the medical conditions and other sleep disorders of the elderly, which can also cause insomnia. For example, health issues such as gastrointestinal and respiratory problems can disrupt sleep. Sleep apnea - in which a person briefly but repeatedly stops breathing during sleep - can also cause insomnia.

According to Vanlint and Jacobo Wartzman, obese subjects had significantly lower basal 25-hydroxyvitamin D concentrations compared to non-obese, which correlated with our results [14,15]. With increasing body mass index (BMI), a decrease in Vitamin D serum levels was observed in our study samples. 12 patients were in (Fig. 4) the category of underweight, i.e., <18.5 BMI, 11 patients belonged to normal weight category and 17 and 19 patients under overweight and obese categories, respectively. This is because Vitamin D is fat-soluble and is readily stored in adipose tissue, it could be sequestered in the larger body pool of fat in obese individuals. It can also be due to lower dietary intake, reduced activation, or increased catabolism. Another possibility is that obese individuals usually follow a sedentary lifestyle which rarely exposes them to sunlight than non-obese individuals, resulting in reduced synthesis of Vitamin D. However, these findings were in contrast with the findings of Zam where he explained obesity to be inversely related to Vitamin D level [16].

According to Li et al. and David et al., insomnia-induced hypertension is very common. This is similar to our study where the insomniacs with

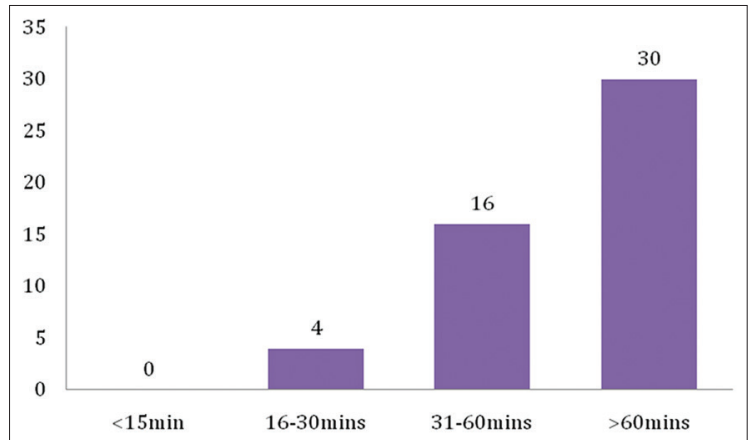

Fig. 7: Time taken to fall asleep by the insomniacs

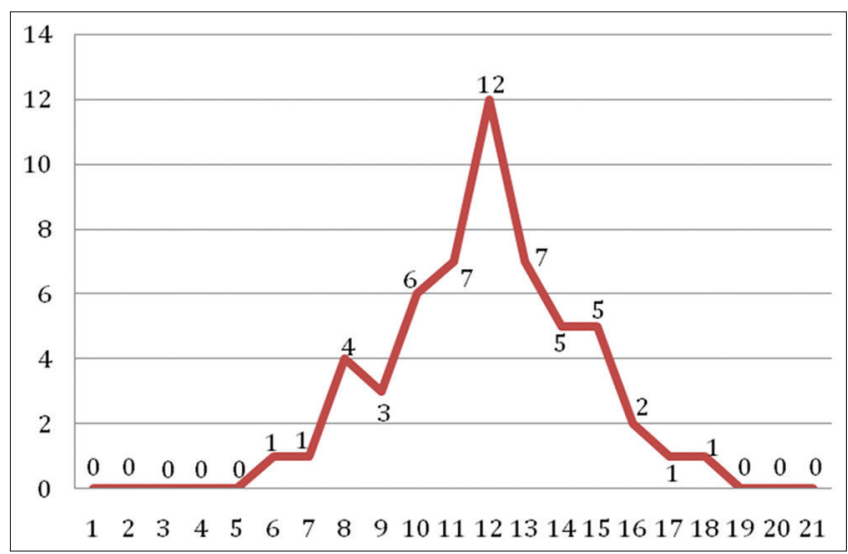

Fig. 8: Marked line graph depicting global Pittsburgh Sleep Quality Index (PSQI) score and the corresponding number of patients. As of the PSQI grading, a score of 5 or $>5$ is indicative of poor sleep quality. All the 50 patients who were assessed had a total PSQI score $>6$, suggesting that all our patients are insomniacs

Table 1: Various causes for trouble sleeping and the number of patients experiencing such problems

\begin{tabular}{ll}
\hline Causes for trouble sleeping & $\begin{array}{l}\text { Number of } \\
\text { patients }\end{array}$ \\
\hline 1. Wake up in the middle of the night or early morning & 50 \\
2. Have to get up to use the bathroom & 50 \\
3. Cough or snore loudly & 08 \\
4. Feel too cold or hot & 07 \\
5. Have bad dreams & 18 \\
6. Have pain & 05 \\
\hline
\end{tabular}

Vitamin D deficiency tend to have increased systolic blood pressure. 27 of 50 patients had a systolic blood pressure of $150 \mathrm{mmHg}$ or greater (Fig. 5). None had a systolic blood pressure $<120 \mathrm{mmHg}$. As concluded in the above-mentioned studies, this is due to the activation of the hypothalamic-pituitary-adrenal axis and the sympathetic nervous system as seen in insomnia $[17,18]$.

Time at which the patients go to sleep was noted, and it is seen that the maximum number of patients goes to sleep by 10:00 PM (11 patients of 50) (Fig. 6). Strangely, a slight increase (8 patients) is seen around 8:00 PM also. Time taken to fell asleep was also noted where most of the patients take more than $1 \mathrm{~h}$ to fell asleep from the time of them going to bed. 30 of our patients took more than $60 \mathrm{~min}$ and 16 of them took 31-60 min, 4 patients between 16 and $30 \mathrm{~min}$, and none fall asleep $<15 \mathrm{~min}$. The delayed time to go to bed and the increased time to fell asleep can be again correlated with the use of mobile phones among young adult during bedtime. 


\section{CONCLUSION}

Our results concluded that insomnia has a direct link with Vitamin D deficiency. Male insomniacs who were Vitamin D deficient were 40\% more than female insomniacs with Vitamin D deficiency. It was noted that the incidence of increased BMI and systolic blood pressure was seen among Vitamin D-deficient insomniacs. Insomnia was more prevalent among younger age groups rather than older adults. Global PSQI value evaluated among all Vitamin D-deficient patients was $>5$, which indicated poor sleep quality.

\section{AUTHORS' CONTRIBUTIONS}

Maria Jai Joseph contributed in collecting personal information and laboratory data of the patients considered in this study which was followed by data analysis. Dr. V.P Karthik contributed in choosing the topic, guided throughout the progress of the study, and cross-checked the study on completion.

\section{CONFLICTS OF INTEREST}

There are no conflicts of interest between the authors.

\section{REFERENCES}

1. Talluri M, Saha S, Adahalli SB. Development and evaluation of sublingual tablet of zolpidem tartrate an antipsychotic drug. Int J Pharm Pharm Sci 2016;8:179-87.

2. Li Y, Yang Y, Li Q, Yang X, Wang Y, Ku WL, et al. The impact of the improvement of insomnia on blood pressure in hypertensive patients. J Sleep Res 2017;26:105-14

3. Jessica A, Ambika A. Role of Vitamin D in insulin secretion and insulin sensitivity for glucose homeostasis. Int J Endocrinol 2009;2010:1-18.

4. Esra T, Babak M, Eve V. Obstructive sleep apnea and Type 2 diabetes: Interacting epidemics. Chest 2008;133:496-506.

5. Early Release of Selected Estimates Based on Data from the January-
June 2003 National Health Interview Survey Released 12/17/2003. Available from: http://www.cdc.gov/nchs/about/major/nhis/released 200312.htm14. [Last cited on 2005 Jan 20].

6. Vgontzas AN, Zoumakis E, Lin HM, Bixler EO, Trakada G, Chrousos GP, et al. Marked decrease in sleepiness in patients with sleep apnea by etanercept, a tumor necrosis factor-alpha antagonist. J Clin Endocrinol Metab 2004;89:4409-13.

7. Buysee DJ, Reynolds CF, Monk TH, Berman SR, Kupfer DJ. The Pittsburgh sleep quality index: A new instrument for psychiatry practice and research. Psychiatr Res 1989;28:198-213

8. Olutayo OA, Abiodun OA, Bola AO, Boladale MM. Validity of the Pittsburgh sleep quality index (PSQI) among Nigerian university students. Sleep Med 2007;8:266-70.

9. Kuningas M, Mooijaart SP, Jolles J, Slagboom P, Westendrop RG, Heemstd VD. Gene variants associated with cognitive function and depressive symptoms in old age. Neurobiol Aging 2009;30:466-73.

10. McCarty DE. Resolution of hypersomnia following identification and treatment of Vitamin D deficiency. J Clin Sleep Med 2010;6:605-8.

11. Zhang Y, Cifuentes M, Gao X, Amaral G, Tucker KL. Age-and genderspecific associations between insomnia and falls in Boston Puerto Rican adults. Qual Life Res 2017;26:25-34

12. Vanlint S. Vitamin D and obesity. Nutrients 2013;5:949-56.

13. Zam W. Vitamin D deficiency and depressive disorders: Review study of probable relationship. Int J Pharm Pharm Sci 2016;8:16-22.

14. Matsuoka LY, Chen TC, Lu Z, Holick MF. Decreased bioavailability of vitamin D in obesity. Am J Clin Nutr 2000;72:690-3.

15. McCann JC, Ames BN. Is there convincing biological or behavioral evidence linking Vitamin D deficiency to brain dysfunction? FASHB J 2008;22:982-1001.

16. Verdoia M, Schaffer A, Sartori C, Barbieri L, Cassetti E, Marino P, et al. Vitamin D deficiency is independently associated with the extent of coronary artery disease. Eur J Clin Invest 2014;44:634-42.

17. Roth T. Insomnia: Definition, prevalence, etiology, and consequences. J Clin Sleep Med 2007;3:S7-10.

18. Sue J, Joanne K, Mary B, Carol EF. Vitamin D and depression: Where is all the sunshine? Mental Health Nurs 2010;31:385-93. 